\title{
The use of mindfulness-based stress reduction (MBSR) for lung cancer patients: protocol for a systematic review and meta-analysis
}

\author{
Xu Tian ${ }^{1,2 \#}$, Zhao-Li Zhang ${ }^{2 \#}$, Yan-Fei Jin ${ }^{1,3}$, Hui Chen $^{2}$, Maria F. Jiménez-Herrera ${ }^{1 \wedge}$ \\ ${ }^{1}$ Nursing Department, Universitat Rovira i Virgili, Tarragona, Spain; ${ }^{2}$ Chongqing University Cancer Hospital, Chongqing, China; ${ }^{3}$ Xiangya School \\ of Nursing of Central South University, Changsha, China \\ Contributions: (I) Conception and design: X Tian, MF Jiménez-Herrera; (II) Administrative support: XL Liu, MF Jiménez-Herrera; (III) Provision of \\ study materials or patients: X Tian; (IV) Collection and assembly of data: X Tian; (V) Data analysis and interpretation: X Tian, YF Jin, H Chen; (VI) \\ Manuscript writing: All authors; (VII) Final approval of manuscript: All authors. \\ \#These authors contributed equally to this work. \\ Correspondence to: Dr. Maria F. Jiménez-Herrera. Nursing Department, Universitat Rovira i Virgili, Tarragona, Spain. Email: maria.jimenez@urv.cat.
}

Background: Mindfulness-based stress reduction (MBSR) intervention has been extensively applied in
cancer patients for relieving symptom burden and its effectiveness has also been demonstrated. However,
the effectiveness of MBSR on psychological and physical functions in lung cancer patients has not yet been
determined. The aim of the present systematic review and meta-analysis seeks to determine the role of
MBSR in lung cancer patients.
Methods: A systematic search of PubMed, EMBASE, Cochrane Library, and China National
Knowledgement Infrastructure (CNKI) will be carried out from their inception until to December 30,
2020. Studies investigating the comparative effects between MBSR and control groups on psychological
and physical outcomes will be documented. Data concerning studies, patient characteristics, and outcomes
will be extracted. Methodological quality of each eligible randomized controlled trial (RCT) will be assessed
individually by two investigators independently using criteria recommended in the Cochrane Handbook for
Systematic Reviews of Interventions 5.1 .0 . Meanwhile, Newcastle-Ottawa Quality Assessment Scale (NOS)
will be used to assess methodological quality of non-randomized studies. All statistical analyses will be
performed with RevMan and STATA softwares.
Discussion: The role of MBSR in lung cancer patients has not yet been demonstrated. This systematic
review and meta-analysis will further determine the effectiveness of MBSR on psychological and physical
outcomes and QoL among lung cancer patients, which will provide golden references for developing
psychological interventions in order to improve patient care and designing future studies to bridge the gap
between research findings and clinical practice.

Trial registration: We registered the protocol of this systematic review and meta-analysis in Open Science Framework (OSF) platform with a registration DOI of 10.17605/OSF.IO/MWVBQ (available from: https:// osf.io/mwvbq).

Keywords: Lung cancer; psychological function; physical function; protocol; systematic review

Submitted Jan 24, 2021. Accepted for publication Mar 31, 2021.

doi: 10.21037/apm-21-194

View this article at: http://dx.doi.org/10.21037/apm-21-194

^ ORCID: Xu Tian, 0000-0002-3275-8751; Maria F. Jiménez-Herrera, 0000-0003-2599-3742. 


\section{Introduction}

Lung cancer is the second most common type of cancer worldwide and also the first-rank reason of causing cancerrelated death (1). The increasing incidence of lung cancer can be attributed to numerous aspects, such as aging of population and environmental pollution (2). It is reported that the new cases of lung cancer have been more than 2.20 million, and approximately 1.80 million deaths in 2020 have been estimated (1).

Although a series of treatment modalities such as surgical operation, chemotherapy, radiotherapy, immuno-biological cancer therapy, and complementary and alternative therapy have been used for treating lung cancer (3-5), patients remain experiencing many serious symptoms such as fatigue (6) and symptom distress (7) because all treatments have also destructive effects on both cancer and normal cells (8). Moreover, cancer self also play a critical role in the development of symptoms $(9,10)$. Previous studies revealed that lung cancer patients experienced more symptom burden than patients who were diagnosed with other types of cancer $(7,9,11)$. It is acknowledged that these all symptoms are significantly associated with reduced psychosocial and physical functions among lung cancer patients $(8,12)$. Additionally, the negative association between symptoms and quality of life (QoL) has also been established by several studies (13-15).

Previous findings have evidently established the effects of psychosocial interventions in improving psychological and physical wellbeing and QoL of cancer patients (16-18). As one of the most common psychosocial interventions, mindfulness-based stress reduction (MBSR) has been extensively applied in cancer patients $(18,19)$. MBSR program was initially developed by Kabat-Zinn to consist of an 8-week psycho-educational program and four meditative techniques including sitting meditation, body scan, gentle Hatha yoga, and walking meditation (20-22). Training of MBSR will make participants gradually to be out of dysfunctional thoughts and directly perceive the emotions and bodily sensations at the present moment via repeatedly bring participants' attention back to the present moment (23). As a result, MBSR program enable participants to step back from ruminating about the past or worrying about the future and simply unfold experiences of the present moment (21). MBSR has been demonstrated to be effective in relieving symptom burden and improving psychosocial adjustment to disease and QoL to date $(18,19,24,25)$. Some studies also tried to investigate the effects of MBSR in lung cancer patients $(26,27)$ and other lung diseases such as interstitial lung diseases $(28,29)$. However, the systematic review and meta-analysis specifically for lung cancer patients is greatly limited except for one meta-analysis which investigated the effects of MBSR on fatigue among lung cancer with subgroup analysis (24).

Therefore, we conduct the current systematic review and meta-analysis to comprehensively evaluate the benefits of MBSR on psychological and physical functions and QoL in patients with lung cancer. We present the following article in accordance with the PRISMA reporting checklist (available at http://dx.doi.org/10.21037/apm-21-194) (30).

\section{Methods}

We designed the methodological framework of this systematic review and meta-analysis protocol in accordance with the methodology recommended by the Cochrane handbook (31). Additionally, we will report our findings in line with the Preferred Reporting Items for Systematic Reviews and Meta-Analyses (PRISMA) guidelines (32). No ethics approval and informed consent will be required because all statistical analysis will be performed based on published studies without involvement of patients. The protocol of this systematic review and meta-analysis has been registered in Open Science Framework (OSF) platform on 22 January, 2021. A registration DOI of 10.17605/OSF.IO/MWVBQ has been approved (available from: https://osf.io/mwvbq).

\section{Search strategy}

We will electronically search PubMed, EMBASE, Cochrane Library, and China National Knowledgement Infrastructure (CNKI) for potentially eligible studies from their inception to December 30, 2020. We will construct the search strategy with the combination of medical subject heading $(\mathrm{MeSH})$ terms and text words. We will verify the systematic review and meta-analysis which also focused our topic and the bibliographies of eligible studies in order to add any additionally eligible study. As an example, we summarized the search strategy of PubMed in Table 1. The process of identification and selection of literature will be displayed in Figure 1. Any disagreement about retrieval of eligible studies will be settled through consulting a third senior author.

\section{Study selection}

One study will be included in the current systematic review 
Table 1 Search strategy of PubMed

\begin{tabular}{ll}
\hline Search number & Query \\
\hline 7 & \#3 AND \#6 \\
6 & \#4 OR \#5 \\
5 & $((((($ Pulmonary Neoplasms[Title/Abstract]) OR (Lung Neoplasm[Title/Abstract])) OR (Pulmonary Neoplasm[Title/ \\
& Abstract])) OR (Lung Cancer[Title/Abstract])) OR (Lung Cancers[Title/Abstract])) OR (Pulmonary Cancer[Title/Abstract])) \\
& OR (Pulmonary Cancers[Title/Abstract]) \\
4 & "Lung Neoplasms"[Mesh] \\
3 & \#1 OR \#2 \\
2 & $(((($ mindfulness[Title/Abstract]) OR (mindfulness meditation[Title/Abstract])) OR (mindfulness-based stress \\
& reduction[Title/Abstract])) OR (mindfulness based stress reduction[Title/Abstract])) OR (MBSR[Title/Abstract]) \\
& "Mindfulness"[Mesh] \\
\hline
\end{tabular}

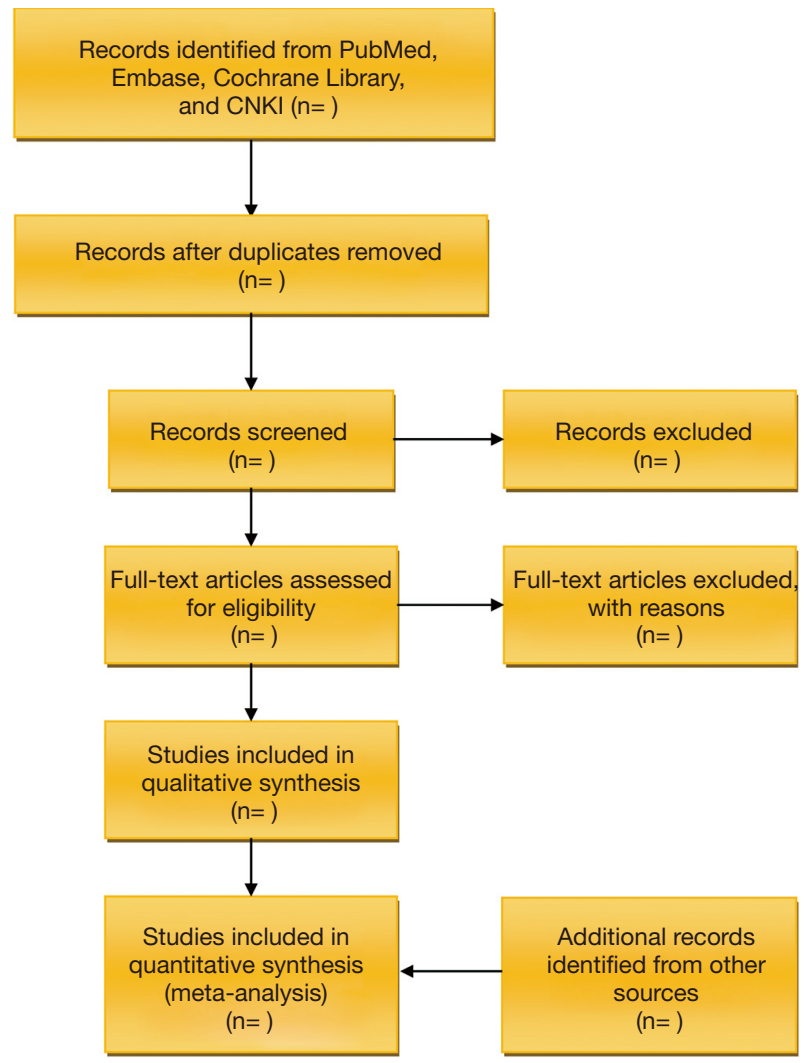

Figure 1 The process of identification and selection of literature.

and meta-analysis if the following criteria were covered: (I) patients, with the age of 18 years or older and irrespective of gender, were diagnosed with lung cancer; (II) MBSR in research group; (III) usual care (UC) or no intervention in control group; (IV) psychological and physical outcomes such as self-designed efficacy and cancer-related fatigue which were defined as primary outcomes and QOL which was defined as secondary outcome; (V) clinical controlled trial (CCT) and randomized clinical trial (RCT); (VI) only full text published in English and Chinese language will be eligible for our inclusion criteria.

One study will be excluded if it covered at least one of the following criteria: (I) MBSR was not used in research group; (II) included mixed patients; and (III) protocol, unpublished or duplicate without adequate information or poor quality.

\section{Data extraction}

Two authors will be assigned to independently extract essential data with designed information extraction form (Table 2). The following information will be extracted from eligible studies: basic information eligible study including author, publication year and country, basic information of patients including sample size, age and tumor staging such as TNM stage, basic information of regimes including details of interventions, outcomes and study design, and information of methodological quality. Quantitative data will be extracted to estimate effect sizes. Data on effect size that could not be obtained directly will be recalculated when possible. Qualitative information will also be summarized to systematically describe the effects and safety of MBSR. Any disagreement about data extraction will be resolved through consulting a third senior author.

\section{Metbodological quality assessment}

Two authors will be assigned to independently assess the methodological quality of eligible studies. Methodological quality of individual RCT will be appraised with the 
Table 2 Basic characteristics of all eligible studies

\begin{tabular}{|c|c|c|c|c|c|c|c|c|c|}
\hline \multirow{2}{*}{ Study } & \multirow{2}{*}{ Country } & \multirow{2}{*}{ Sample size } & \multirow{2}{*}{ Age, year } & \multicolumn{2}{|c|}{ Interventions } & \multirow{2}{*}{ Follow-up } & \multirow{2}{*}{ Outcomes } & \multirow{2}{*}{ Instruments } & \multirow{2}{*}{ Study design } \\
\hline & & & & $R G$ & CG & & & & \\
\hline
\end{tabular}

Study 1

Study 2

Study 3

Cochrane risk of bias tool as following six domains including (33): (I) random sequence generation; (II) allocation concealment; (III) blinding of personnel, participants, and assessors; (IV) incomplete outcome data; (V) selective reporting; and (VI) other bias. Additionally, methodological quality of nonrandomized studies will be assessed with Newcastle-Ottawa Quality Assessment Scale (NOS) from three aspects: selection of study groups, comparability of study groups and ascertainment of either the exposure or outcome of interest (34). Any disagreement about the methodological quality assessment will be resolved through consulting a third senior author.

\section{Statistical analysis}

For continuous outcomes, we will calculate the differences between two groups as the standard mean difference (SMD) or mean difference (MD) with $95 \%$ confidence interval (CI). For categorical outcomes, we will use risk ratio (RR) with 95\% CI to express pooled estimates. In this meta-analysis, we only extract immediate, post-intervention data (after a 6-16 week course) to estimate the effect size in order on achieve uniformity according to the methods used in previous meta-analysis (17). Meanwhile, we also extract the data at the first time point which is defined as immediately post-intervention for statistical analysis if a study reported data on a series of time points. Before performing statistical analysis, we will use Cochrane's Q test and the $\mathrm{I}^{2}$ statistic to qualitatively and quantitatively evaluate the heterogeneity across studies accordingly $(35,36)$. Nevertheless, we will only perform a random-effects model to calculate all pooled results regardless of actual level of heterogeneity across studies because substantial variations in population, interventions, and outcome measures are inevitable. A $\mathrm{P}<0.05$ will be considered to be the criteria of statistical significance. All statistical analyses will be conducted using Review Manager (RevMan) version 5.3 (Cochrane
Collaboration, Oxford, United Kingdom).

\section{Publication bias}

For individual outcome, we will draw funnel plot and perform Egger's tests respectively to test the possibility of publication bias when accumulated number of 10 were achieved (37-39). STATA 14.0 software (StataCorp, Texas, USA) will be utilized to complete Egger's tests.

\section{Quality of evidence assessment}

We will use the Grading of Recommendations Assessment, Development and Evaluation method (40) to grade the overall quality of evidence of quantitative pooled results as high, moderate, low, or very low after completing all statistical analyses. With the GRADE system, the level of RCT will be initially rated as high, and the level can be downgraded according to five aspects including risk of bias, inconsistency, indirectness, imprecision, and publication bias. Two authors will independently rate the level of evidence and any disagreement will be resolved based on the consensus principle.

\section{Discussion}

As one of the most common malignant tumors around the world, lung cancer was estimated to account for $11.4 \%$ new cancer cases and $18.0 \%$ cancer deaths in 2020 (41). Evidence suggested lung cancer patients will experience a series of serious symptoms due to the negative impact of definitive diagnosis of cancer and anticancer treatments $(7,9)$. As a result, lung cancer patients will also suffer from poor psychological and physical functions and QoL $(6,7)$. As one of the most common psychological intervention programs, MBSR have been extensively investigated and also established to improve symptoms of cancer patients 
$(19,24,26)$. However, there was no systematic review and meta-analysis specifically focused on the impacts of MBSR intervention on psychological and physical functions and QoL of lung cancer patients has been reported to date. As a result, it remains unclear whether MBSR should be preferentially prescribed for the treatment of psychological and physical conditions among lung cancer patients. The present systematic review and meta-analysis will provide a comprehensive summary of studies exploring the effectiveness of MBSR on psychological and physical outcomes and QoL in lung cancer patients.

To our knowledge, this is the first systematic review and meta-analysis of RCTs and CCTs that will investigate the effects of MBSR on psychological and physical outcomes and QoL for lung cancer patients. However, we also must further interpret the limitations in this systematic review and meta-analysis. First, we will only search four databases including PubMed, EMBASE, Cochrane Library and CNKI, and thus some potentially eligible studies included in other databases such as Web of Science and SCOPUS may be missed. Second, the substantial variations in intensity, frequency, and duration of MBSR across studies may introduce heterogeneity, we thus use a random-effects model to perform all statistical analyses, which will result in wider $95 \%$ CI. Third, definitions of usual care in individual study are different, however subgroup analysis will not be designed because details of usual care were not introduced.

\section{Conclusions}

The systematic review and meta-analysis will investigate the effectiveness of MBSR intervention on psychological and physical functions and QoL in patients with lung cancer. The results from the current study will demonstrate the effects of MBSR on specific outcomes and then provide evidence for developing psychological interventions in clinical practice. Meanwhile, this study also determines the gap between research findings and clinical practice, and then provides references for designing further studies.

\section{Acknowledgments}

We express our sincere appreciation to all authors who participated in this study.

Funding: This study has been supported by grants from the Technological Innovation and Demonstrational Application Project of Chongqing Science and Technology Bureau (project No. cstc2018jscx-msybX0030) and
Chongqing Natural Science Foundation (project no. cstc2018jcyjAX0737s).

\section{Footnote}

Reporting Checklist: The authors have completed the PRISMA reporting checklist. Available at http://dx.doi. org/10.21037/apm-21-194

Conflicts of Interest: All authors have completed the ICMJE uniform disclosure form (available at http://dx.doi. org/10.21037/apm-21-194). Dr. XT reports grants from the Technological Innovation and Demonstrational Application Project of Chongqing Science and Technology Bureau (project No. cstc2018jscx-msybX0030) and Chongqing Natural Science Foundation (project No. cstc2018jcyjAX0737s). The other authors have No conflicts of interest to declare.

Ethical Statement: The authors are accountable for all aspects of the work in ensuring that questions related to the accuracy or integrity of any part of the work are appropriately investigated and resolved.

Open Access Statement: This is an Open Access article distributed in accordance with the Creative Commons Attribution-NonCommercial-NoDerivs 4.0 International License (CC BY-NC-ND 4.0), which permits the noncommercial replication and distribution of the article with the strict proviso that no changes or edits are made and the original work is properly cited (including links to both the formal publication through the relevant DOI and the license). See: https://creativecommons.org/licenses/by-nc-nd/4.0/.

\section{References}

1. Sung H, Ferlay J, Siegel RL, et al. Global cancer statistics 2020: GLOBOCAN estimates of incidence and mortality worldwide for 36 cancers in 185 countries. CA Cancer J Clin 2021;71:209-49.

2. Mao Y, Yang D, He J, et al. Epidemiology of Lung Cancer. Surg Oncol Clin N Am 2016;25:439-45.

3. Gadgeel SM, Ramalingam SS, Kalemkerian GP. Treatment of lung cancer. Radiol Clin North Am 2012;50:961-74.

4. Jurisevic M, Bolevich S. Complementary and alternative medicine applications in cancer medicine. Traditional Medicine Research 2020;5:1-61.

5. Yang X, Luan X. Annual advances in traditional medicine 
for tumor therapy in 2019. Traditional Medicine Research 2020;5:62-121.

6. Iyer S, Taylor-Stokes G, Roughley A. Symptom burden and quality of life in advanced non-small cell lung cancer patients in France and Germany. Lung Cancer 2013;81:288-93.

7. Morrison EJ, Novotny PJ, Sloan JA, et al. Emotional Problems, Quality of Life, and Symptom Burden in Patients With Lung Cancer. Clin Lung Cancer 2017;18:497-503.

8. Yang M, Liu L, Gan CE, et al. Effects of home-based exercise on exercise capacity, symptoms, and quality of life in patients with lung cancer: A meta-analysis. Eur J Oncol Nurs 2020;49:101836.

9. Iyer S, Roughley A, Rider A, et al. The symptom burden of non-small cell lung cancer in the USA: a real-world cross-sectional study. Support Care Cancer 2014;22:181-7.

10. Sung MR, Patel MV, Djalalov S, et al. Evolution of Symptom Burden of Advanced Lung Cancer Over a Decade. Clin Lung Cancer 2017;18:274-80.e6.

11. Chan CW, Chair SY, Chui YY. End of life experience of symptom cluster and their management in Hong Kong chinese patients with lung cancer who receive palliative radiotherapy. Zhongguo Fei Ai Za Zhi 2009;12:361-8.

12. Lee J. Physiologic and psychologic adaptation to exercise interventions in lung cancer patients undergoing chemotherapy: a systematic review and meta-analysis of randomized controlled trials. Support Care Cancer 2021;29:2863-73.

13. Choi S, Ryu E. Effects of symptom clusters and depression on the quality of life in patients with advanced lung cancer. Eur J Cancer Care (Engl) 2018;27.

14. Möller A, Sartipy U. Long-term health-related quality of life following surgery for lung cancer. Eur J Cardiothorac Surg 2012;41:362-7.

15. Park S, Kang CH, Hwang Y, et al. Risk factors for postoperative anxiety and depression after surgical treatment for lung cancer†. Eur J Cardiothorac Surg 2016;49:e16-21.

16. Galway K, Black A, Cantwell M, et al. Psychosocial interventions to improve quality of life and emotional wellbeing for recently diagnosed cancer patients. Cochrane Database Syst Rev 2012;11:CD007064.

17. Huang HP, He M, Wang HY, et al. A meta-analysis of the benefits of mindfulness-based stress reduction (MBSR) on psychological function among breast cancer (BC) survivors. Breast Cancer 2016;23:568-76.

18. Cillessen L, Johannsen M, Speckens AEM, et al.
Mindfulness-based interventions for psychological and physical health outcomes in cancer patients and survivors: A systematic review and meta-analysis of randomized controlled trials. Psychooncology 2019;28:2257-69.

19. Lee CE, Kim S, Kim S, et al. Effects of a MindfulnessBased Stress Reduction Program on the Physical and Psychological Status and Quality of Life in Patients With Metastatic Breast Cancer. Holist Nurs Pract 2017;31:260-9.

20. Kabat-Zinn J, Wheeler E, Light T, et al. Influence of a mindfulness meditation-based stress reduction intervention on rates of skin clearing in patients with moderate to severe psoriasis undergoing phototherapy (UVB) and photochemotherapy (PUVA). Psychosom Med 1998;60:625-32.

21. Kabat-Zinn J, Lipworth L, Burney R. The clinical use of mindfulness meditation for the self-regulation of chronic pain. J Behav Med 1985;8:163-90.

22. Kabat-Zinn J, Massion AO, Kristeller J, et al. Effectiveness of a meditation-based stress reduction program in the treatment of anxiety disorders. Am J Psychiatry 1992;149:936-43.

23. Morgan D. Mindfulness-based cognitive therapy for depression: a new approach to preventing relapse. Psychother Res 2003;13:123-5.

24. Xie C, Dong B, Wang L, et al. Mindfulness-based stress reduction can alleviate cancer- related fatigue: A metaanalysis. J Psychosom Res 2020;130:109916.

25. Xunlin NG, Lau Y, Klainin-Yobas P. The effectiveness of mindfulness-based interventions among cancer patients and survivors: a systematic review and meta-analysis. Support Care Cancer 2020;28:1563-78.

26. van den Hurk DG, Schellekens MP, Molema J, et al. Mindfulness-Based Stress Reduction for lung cancer patients and their partners: Results of a mixed methods pilot study. Palliat Med 2015;29:652-60.

27. Schellekens MPJ, van den Hurk DGM, Prins JB, et al. Mindfulness-based stress reduction added to care as usual for lung cancer patients and/or their partners: A multicentre randomized controlled trial. Psychooncology 2017;26:2118-26.

28. Sgalla G, Cerri S, Ferrari R, et al. Mindfulness-based stress reduction in patients with interstitial lung diseases: a pilot, single-centre observational study on safety and efficacy. BMJ Open Respir Res 2015;2:e000065.

29. Arefnasab Z, Ghanei M, Noorbala AA, et al. Effect of Mindfulness Based Stress Reduction on Quality of Life (SF-36) and Spirometry Parameters, in Chemically 
Pulmonary Injured Veterans. Iran J Public Health 2013;42:1026-33.

30. Shamseer L, Moher D, Clarke M, et al. Preferred reporting items for systematic review and meta-analysis protocols (PRISMA-P) 2015: elaboration and explanation. Bmj 2015;350:g7647.

31. Higgins JPT, Green S. Cochrane Handbook for Systematic Reviews of Interventions Version 5.1.0 [updated March 2011]. The Cochrane Collaboration, 2011 Available online: wwwhandbookcochraneorg

32. Liberati A, Altman DG, Tetzlaff J, et al. The PRISMA statement for reporting systematic reviews and metaanalyses of studies that evaluate healthcare interventions: explanation and elaboration. BMJ 2009;339:b2700.

33. Higgins JP, Altman DG, Gotzsche PC, et al. The Cochrane Collaboration's tool for assessing risk of bias in randomised trials. BMJ 2011;343:d5928.

34. Wells G, Shea B, O'Connell D, et al. The NewcastleOttawa Scale (NOS) for Assessing the Quality of NonRandomized Studies in Meta-Analysis. 2000;.

Cite this article as: Tian $\mathrm{X}$, Zhang ZL, Jin YF, Chen H, Jiménez-Herrera MF. The use of mindfulness-based stress reduction (MBSR) for lung cancer patients: protocol for a systematic review and meta-analysis. Ann Palliat Med 2021;10(7):8276-8282. doi: 10.21037/apm-21-194
35. Higgins JP, Thompson SG. Quantifying heterogeneity in a meta-analysis. Stat Med 2002;21:1539-58.

36. Higgins JP, Thompson SG, Deeks JJ, et al. Measuring inconsistency in meta-analyses. BMJ 2003;327:557-60.

37. Egger M, Davey Smith G, Schneider M, et al. Bias in meta-analysis detected by a simple, graphical test. BMJ 1997;315:629-34.

38. Page MJ, McKenzie JE, Higgins JPT. Tools for assessing risk of reporting biases in studies and syntheses of studies: a systematic review. BMJ Open 2018;8:e019703.

39. Sterne JA, Egger M. Funnel plots for detecting bias in meta-analysis: guidelines on choice of axis. J Clin Epidemiol 2001;54:1046-55.

40. Guyatt GH, Oxman AD, Vist GE, et al. GRADE: an emerging consensus on rating quality of evidence and strength of recommendations. BMJ 2008;336:924-6.

41. Wild CP, Weiderpass E, Stewart BW, editors. World Cancer Report: Cancer Research for Cancer Prevention. Lyon, France: International Agency for Research on Cancer, 2020. 